Article

\title{
On the Exponents of Exponential Dichotomies
}

\author{
Flaviano Battelli ${ }^{1}[\mathbb{D}$ and Michal Fečkan $2,3, * \mathbb{D}$ \\ 1 Department of Industrial Engineering and Mathematics, Marche Polytecnic University, 60121 Ancona, Italy; \\ battelli@dipmat.univpm.it \\ 2 Department of Mathematical Analysis and Numerical Mathematics, Faculty of Mathematics, Physics and \\ Informatics, Comenius University in Bratislava, Mlynská dolina, 84248 Bratislava, Slovakia \\ 3 Mathematical Institute, Slovak Academy of Sciences, Štefánikova 49, 81473 Bratislava, Slovakia \\ * Correspondence: Michal.Feckan@fmph.uniba.sk
}

Received: 22 March 2020; Accepted: 18 April 2020; Published: 23 April 2020

check for updates

\begin{abstract}
An exponential dichotomy is studied for linear differential equations. A constructive method is presented to derive a roughness result for perturbations giving exponents of the dichotomy as well as an estimate of the norm of the difference between the corresponding two dichotomy projections. This roughness result is crucial in developing a Melnikov bifurcation method for either discontinuous or implicit perturbed nonlinear differential equations.
\end{abstract}

Keywords: exponential dichotomy; roughness; asymptotically constant matrices

MSC: 34D09

\section{Introduction}

Exponential dichotomy of a linear system of differential equations is a type of conditional stability that goes back to an idea in Perron [1]. It was revealed to be a very important tool for the study of nonlinear systems because of its roughness. Indeed, it has been used to show the existence of chaotic behaviour in non autonomous perturbations of autonomous nonlinear equations having a homoclinic solution, since transverse intersection of stable and unstable manifolds along a homoclinic solution corresponds to the fact that the linearization of the nonlinear system along it has an exponential dichotomy on $\mathbb{R}$ [2]. Exponential dichotomies are also related with the so called reducibillty. A linear system of differential equations $\dot{x}=A(t) x$ is said to be reducible if there exists an invertible $C^{1}$ matrix $S(t)$ such that the change of variables $x=S(t) y$ transforms the system into a block diagonal system

$$
\dot{y}=\left(\begin{array}{cc}
B_{1}(t) & 0 \\
0 & B_{2}(t)
\end{array}\right) y
$$

In [3], it is proven that a system is reducible if and only if the original system has an exponential or ordinary dichotomy. The difference between the two cases is that in ordinary dichotomy the exponents are equal to zero. Another interesting property is the following (see [3]). The linear system $\dot{x}=A(t) x$ has an exponential dichotomy on $\mathbb{R}_{+}$if and only if for every locally integrable function $f(t), t \in \mathbb{R}_{+}$, such that

$$
\sup _{t \geq 0} \int_{t}^{t+1} f(s) d s<\infty,
$$

the inhomogeneous linear system $\dot{x}=A(t) x+f(t)$ has a bounded solution. Exponential dichotomies have also relations with such notions as integral separation or spectral theory, see for example $[4,5]$. Recently, it has been proved in [6] that if a bounded linear Hamiltonian system is exponentially separated into two subspaces of the same dimension, then it must have an exponential dichotomy. 
Let us start with the definition of exponential dichotomy. A linear system

$$
\dot{x}=A(t) x
$$

where $A(t)$ is a piecewise continuous $n \times n$ matrix, is said to have an exponential dichotomy on an interval $I \subset \mathbb{R}$ (usually $\mathbb{R}, \mathbb{R}_{+}, \mathbb{R}_{-}$) with projection $P$, constant $k \geq 1$ and exponents $\alpha, \beta>0$ if the fundamental matrix $X(t)$ of the Equation $(1)$ (with $X(0)=\mathbb{I})$ satisfies the following conditions:

$$
\begin{array}{ll}
\left|X(t) P X(s)^{-1}\right| \leq k e^{-\alpha(t-s)} & \text { for } s \leq t, s, t \in I \\
\left|X(s)(\mathbb{I}-P) X(t)^{-1}\right| \leq k e^{-\beta(t-s)} & \text { for } s \leq t, s, t \in I .
\end{array}
$$

Here $\mathbb{R}=(-\infty, \infty), \mathbb{R}_{+}=[0, \infty)$ and $\mathbb{R}_{-}=(-\infty, 0]$. It follows immediately from the definition that, if $\alpha^{\prime} \leq \alpha$ and $\beta^{\prime} \leq \beta$ then $\alpha^{\prime}$ and $\beta^{\prime}$ are also exponents of the dichotomy with the same projection $P$ and constant $k$ and also that the linear system (1) has an exponential dichotomy on an interval $J \subseteq I$ if it has one in the interval $I$. Next, from Gronwall inequality it follows that, on a compact interval, the linear system (1) has an exponential dichotomy with any projection $P$ and exponents $\alpha$ and $\beta$ (but the constant may change).

We give few examples of systems having an exponential dichotomy. An autonomous system $\dot{x}=A x$ has an exponential dichotomy on $\mathbb{R}$ if and only if all the eigenvalues of $A$ have nonzero real parts. A periodic system $\dot{x}=A(t) x$ has an exponential dichotomy on $\mathbb{R}$ if and only if all the Floquet exponents have nonzero real parts. A scalar equation $\dot{x}=a(t) x$ has an exponential dichotomy on $I=\mathbb{R}_{+}$or $I=\mathbb{R}_{-}$, if and only if

$$
\liminf _{t-s \rightarrow \infty} \frac{1}{t-s} \int_{s}^{t} a(\tau) d \tau>0 \text { or } \limsup _{t-s \rightarrow \infty} \frac{1}{t-s} \int_{s}^{t} a(\tau) d \tau<0 .
$$

where the limits are taken as $t-s \rightarrow \pm \infty$ in case $I=\mathbb{R}_{ \pm}$respectively.

Suppose the linear system $\dot{x}=A(t) x$ has an exponential dichotomy on $\mathbb{R}_{+}$with exponents $\alpha$ and $\beta$. The result that motivates this paper is the following, see [3] (Proposition 1, p. 34).

Theorem 1 (Roughness). Let $\dot{x}=A(t) x$ have an exponential dichotomy on $\mathbb{R}_{+}$with exponents $\alpha$ and $\beta$. Given $0<\tilde{\alpha}<\alpha$ and $0<\tilde{\beta}<\beta$ there exists $\varepsilon>0$ such that if $B(t)$ is a piecewise continuous matrix such that $\sup _{t \in \mathbb{R}_{+}}|B(t)|<\varepsilon$ then the linear system $\dot{x}=[A(t)+B(t)] x$ has an exponential dichotomy on $\mathbb{R}_{+}$with exponents $\tilde{\alpha}, \tilde{\beta}$ (but the constant may be larger).

As a matter of fact in [3] (Proposition 1, p. 34), an estimate on the size of $\varepsilon$ is also given, showing that, if $\beta=\alpha$ and $\varepsilon<\frac{\alpha}{4 k^{2}}$ then $\dot{x}=[A(t)+B(t)] x$ has an exponential dichotomy on $\mathbb{R}_{+}$with exponent $\alpha-2 k \varepsilon$. So if $\beta=\alpha$ and $\tilde{\alpha}=\tilde{\beta}<\alpha$ we have $\varepsilon=\frac{\alpha-\tilde{\alpha}}{2 k}$. We emphasize the fact that in [7] the assumptions on $B(t)$ have been weakened to obtain a roughness result valid also for unbounded perturbations.

However, the exponents of the dichotomy determine the rate of convergence to zero of bounded solution either at $\infty$ (when the dichotomy is in $\mathbb{R}_{+}$) or at $-\infty$ (when the dichotomy is in $\mathbb{R}_{-}$). Sometimes it becomes important to determine this rate of convergence, and hence the exponents of the dichotomy, for example when studying chaotic behaviour of discontinuous systems [8] or developing Melnikov theory for implicit nonlinear differential equations [9]. As a matter of fact in [8] the following result has been proved.

Theorem 2. Let $\dot{x}=A(t) x$ have an exponential dichotomy on $\mathbb{R}_{+}$with exponents $\alpha, \beta$. Then there exists $\varepsilon>0$ such that if $B(t)$ is a piecewise continuous function such that, for some $T>0, \sup _{t \geq T}|B(t)|<\varepsilon$ and

$$
\int_{\bar{T}}^{\infty}|B(t)| d t<\frac{1}{k}
$$


then the linear system $\dot{x}=[A(t)+B(t)] x$ has an exponential dichotomy on $[T, \infty)$ (and hence also on $\mathbb{R}_{+}$) with the same exponents $\alpha, \beta$.

Of course Theorems 1 and 2 hold equally well when the dichotomy of $\dot{x}=A(t) x$ is on $\mathbb{R}_{-}$.

The proof given in [8] follows an idea in [3] where an exponential estimate is derived for bounded solutions of certain integral inequalities. In this paper we want to give another, more direct, proof of the same result. As a matter of fact we work directly in the space of continuous functions decaying to zero as $t \rightarrow \infty$ at a certain given rate. This approach leads us to derive the first of the two exponential estimates given in (2). The second is derived passing to the adjoint system and using the fact that one has a certain freedom in choosing the projection of the dichotomy (see Proposition 2).

Our method has also the advantage that relates the projection of the dichotomy of the perturbed system with the one of the unperturbed. As a matter of fact, we will give an estimate of the norm of the difference between the two projections in term of $\sup _{t \in I}|B(t)|$, where $I=\mathbb{R}_{+}, \mathbb{R}_{-}$is the interval where the exponential dichotomy is considered. This estimate allows us to prove the same result also when the dichotomy of the unperturbed system is on $\mathbb{R}$, a fact that was not noted in [8].

We now briefly resume the content of this paper. In Section 2 we recall basic properties of exponential dichotomy, stable and unstable spaces, roughness, freedom in the choice of the projection etc. Section 3 is devoted to the proof of our main result. Finally, Section 4 contains applications to asymptotically constant matrices and to the linearization of nonlinear systems.

We conclude this section by giving some notations used in the paper. For a linear map $L$ from a Banach space into another, we denote by $\mathcal{R} L$ and $\mathcal{N} L$ its range, resp. its kernel. Next $C_{b}^{0}(I)$ denotes the Banach space of bounded continuous functions $x(t)$ on the interval $I$ with the norm $\|x\|=\sup _{t \in I}|x(t)|$. When $I=\mathbb{R}_{+}$or $\mathbb{R}_{-}$we omit $I$ and write $C_{b}^{0}$ instead of $C_{b}^{0}\left(\mathbb{R}_{+}\right)$or $C_{b}^{0}\left(\mathbb{R}_{-}\right)$.

\section{Properties of Exponential Dichotomies}

First we start with a remark. Let $v \in \mathbb{R}$ be a real number. Then $Y(t)=X(t) e^{v t}$ is a fundamental matrix of the linear system

$$
\dot{x}=[A(t)+v \mathbb{I}] x .
$$

Assuming that (1) has an exponential dichotomy on $I$ with exponents $\alpha, \beta$, we have, for $s, t \in I$, with $s \leq t$ :

$$
\begin{aligned}
& \left|Y(t) P Y(s)^{-1}\right| \leq k e^{-(\alpha-v)(t-s)} \\
& \left|Y(s)(\mathbb{I}-P) Y(t)^{-1}\right| \leq k e^{-(\beta+v)(t-s)}
\end{aligned}
$$

that is (3) has an exponential dichotomy on $I$ with the same projection $P$, constant $k$ and exponents $(\alpha-v)$ and $(\beta+v)$. Viceversa, if (3) has an exponential dichotomy on $I$ with projections $P$, constant $k$ and exponents $\tilde{\alpha}, \tilde{\beta}$, then (1) has an exponential dichotomy on $I$ with the same projections $P$ and constant $k$, and exponents $\alpha=\tilde{\alpha}+v, \beta=\tilde{\beta}-v$. Taking $v=\frac{\alpha-\beta}{2}$ the exponents of the dichotomy of (3) are then

$$
\tilde{\alpha}=\alpha-\frac{\alpha-\beta}{2}=\frac{\alpha+\beta}{2}=\beta+\frac{\alpha-\beta}{2}=\tilde{\beta} .
$$

So, starting from a linear system with an exponential dichotomy, shifting the coefficient matrix by $v \mathbb{I}, v=\frac{\alpha-\beta}{2}$, we can assume that the exponents are the same.

Proposition 1. Suppose that (1) has an exponential dichotomy of the intervals $I_{1}$ and $I_{2}$ with the same projection and exponents. Suppose, also, that $I_{1} \cap I_{2} \neq \varnothing$. Then (1) has an exponential dichotomy of the interval $I_{1} \cup I_{2}$ with the same projection and exponents but possibly different constant.

Proof. If $I_{1} \subset I_{2}$ or $I_{2} \subset I_{1}$ there is nothing to prove. So we assume that $I=I_{1} \cap I_{2}$ is different from both $I_{1}$ and $I_{2}$. We can also assume that $I_{1}$ is on the left and $I_{2}$ is on the right that is: if $t_{1} \in I_{1} \backslash I$ and $t_{2} \in I_{2} \backslash I$ then $t_{1}<t_{2}$. 
It is clear that (2) holds if $s, t \in I_{1}$ or $s, t \in I_{2}$. So, let $s \in I_{1} \backslash I$ and $t \in I_{2} \backslash I$. Take $\bar{t} \in I$. Then $s<\bar{t}<t$ and we have:

$$
\begin{aligned}
& \left|X(t) P X(s)^{-1}\right| \leq\left|X(t) P X(\bar{t})^{-1}\right|\left|X(\bar{t}) P X(s)^{-1}\right| \\
& \quad \leq k e^{-\alpha(t-\bar{t})} k e^{-\alpha(\bar{t}-s)}=k^{2} e^{-\alpha(t-s)} \\
& \left|X(s)(I-P) X(t)^{-1}\right| \leq\left|X(s)(\mathbb{I}-P) X(\bar{t})^{-1}\right|\left|X(\bar{t})(\mathbb{I}-P) X(t)^{-1}\right| \\
& \quad \leq k e^{\beta(s-\bar{t})} k e^{\beta(\bar{t}-t)}=k^{2} e^{\beta(s-t)}
\end{aligned}
$$

the proof is complete.

Since in compact intervals $I=[a, b]$ a linear system (1) has an exponential dichotomy with any projection and any exponents, it follows from Proposition 1 that if a linear system has an exponential dichotomy on an interval $[T, \infty)$ (resp. $(-\infty,-T])$ then it has an exponential dichotomy with the same exponents and projection on $\mathbb{R}_{+}=[0, \infty)$ (resp. $\left.\mathbb{R}_{-}=(-\infty, 0]\right)$. Hence, in the following we will only consider $I=\mathbb{R}_{+}$or $I=\mathbb{R}_{-}$.

When the dichotomy is on $\mathbb{R}_{+}$(or on $\mathbb{R}_{-}$) we have some freedom in the choice of the projection. Indeed we have the following

Proposition 2. [3] (p. 16-17). Suppose (1) has an exponential dichotomy on $\mathbb{R}_{+}$with projection $P$. Then

$$
\mathcal{R} P=\left\{\xi \in \mathbb{R}^{n}: \sup _{t \geq 0}|X(t) \xi| e^{\alpha t}<\infty\right\}=\left\{\xi \in \mathbb{R}^{n}: \sup _{t \geq 0}|X(t) \xi|<\infty\right\}
$$

but the kernel of $P, \mathcal{N} P$, can be any complement of $\mathcal{R} P$. Moreover if $Q: \mathbb{R}^{n} \rightarrow \mathbb{R}^{n}$ is another projection such that $\mathcal{R} Q=\mathcal{R} P$ then there exist a constant $k_{Q}$ such that (2) holds with $Q$ and $k_{Q}$ instead of $P$ and $k$ (with the same exponents). If the dichotomy is on $\mathbb{R}_{-}$then it is $\mathcal{N} P$ which is uniquely defined being

$$
\mathcal{N} P=\left\{\xi \in \mathbb{R}^{n}: \sup _{t \leq 0}|X(t) \xi| e^{-\beta t}<\infty\right\}=\left\{\xi \in \mathbb{R}^{n}: \sup _{t \leq 0}|X(t) \xi|<\infty\right\}
$$

Moreover $\mathcal{R} P$ can be any complement of $\mathcal{N} P$ and if $Q: \mathbb{R}^{n} \rightarrow \mathbb{R}^{n}$ is another projection such that $\mathcal{N} Q=\mathcal{N} P$ then there exist a constant $k_{Q}$ such that (2) holds with $Q$ and $k_{Q}$ instead of $P$ and $k$ (with the same exponents).

A consequence of the roughness Theorem 1 is the following.

Corollary 1. Suppose the linear system (1) has an exponential dichotomy on $\mathbb{R}_{+}\left[\right.$resp. $\left.\mathbb{R}_{-}\right]$with projection $P$ and exponents $\alpha$ and $\beta$. Let $B(t)$ be a matrix such that

$$
\lim _{t \rightarrow \infty}|B(t)|=0
$$

where the limit is taken at $+\infty$ if $I=\mathbb{R}_{+}$and at $-\infty$ when $I=\mathbb{R}_{-}$. Then, given $\tilde{\alpha}<\alpha$ and $\tilde{\beta}<\beta$, the linear system $\dot{x}=[A(t)+B(t)] x$ has an exponential dichotomy on $\mathbb{R}_{+}$[resp. $\left.\mathbb{R}_{-}\right]$with exponent $\tilde{\alpha}$ and $\tilde{\beta}$ and projection $\tilde{P}$ such that $\mathcal{N} \tilde{P}=\mathcal{N} P[$ resp. $\mathcal{R} \tilde{P}=\mathcal{R} P]$.

Proof. Let $\tilde{\alpha}$ and $\tilde{\beta}$ be as in the statement of the theorem and let $\varepsilon>0$ be as in Theorem 1 . It follows from the assumption the existence of $T$ such that for $t \geq T$ we have $|B(t)| \leq \varepsilon$ and the linear system $\dot{x}=A(t) x$ has an exponential dichotomy on $[T,+\infty)$ with projection $P$ and exponents $\alpha$ and $\beta$. Then from Theorem 1 it follows that $\dot{x}=[A(t)+B(t)] x$ has an exponential dichotomy on $[T, \infty)$ with exponent $\tilde{\alpha}$ and $\tilde{\beta}$ and projection as in the statement of the Corollary. However, we have already observed that on $[0, T], \dot{x}=[A(t)+B(t)] x$ has an exponential dichotomy with the same projection and exponents. Then the conclusion follows from Proposition 1. 
Example. Consider the scalar equation $\dot{x}=\left(-1+\frac{1}{t+1}\right) x$. The unperturbed equation $\dot{x}=-x$ has an exponential dichotomy on $\mathbb{R}$ (and hence on both $\mathbb{R}_{+}$and $\mathbb{R}_{-}$) with $k=1, \alpha=1$ and projection $P=\mathbb{I}$. The solution of the perturbed equation with $x(0)=1$ is $x(t)=(t+1) e^{-t}$ and

$$
\left|\frac{x(t)}{x(s)}\right|=\frac{t+1}{s+1} e^{-(t-s)} .
$$

Let $\alpha<1$. The function $(t+1) e^{-(1-\alpha) t}$ is increasing on $\left[0, \frac{\alpha}{1-\alpha}\right]$ and decreasing on $\left[\frac{\alpha}{1-\alpha}, \infty\right)$ hence

$$
(t-s+1) e^{-(1-\alpha)(t-s)} \leq \frac{e^{-\alpha}}{1-\alpha}
$$

for any $s \leq t$. Next, observe that for $0 \leq s \leq t$ we have

$$
\frac{1}{s+1} \leq 1 \Leftrightarrow \frac{t-s}{s+1} \leq t-s \Leftrightarrow \frac{t+1}{s+1} \leq t-s+1
$$

hence

$$
\frac{t+1}{s+1} e^{-(t-s)} \leq \frac{e^{-\alpha}}{1-\alpha} e^{-\alpha(t-s)} .
$$

So the equation $\dot{x}=\left(-1+\frac{1}{t+1}\right) x$ has an exponential dichotomy on $\mathbb{R}_{+}$with exponent $\alpha<1$ but not with exponent $=1$ since otherwise there should exists $k \geq 1$ such that

$$
\frac{t+1}{s+1} \leq k
$$

for any $0 \leq s \leq t$ which is absurd. However, the fundamental solution of scalar equation $\dot{x}=$ $\left(-1+\frac{1}{t^{2}+1}\right) x$ is

$$
x(t)=e^{-t+\arctan t}
$$

and

$$
\left|\frac{x(t)}{x(s)}\right|=\frac{e^{\arctan t}}{e^{\arctan s}} e^{-(t-s)} \leq e^{\frac{\pi}{2}} e^{-(t-s)} .
$$

for any $0 \leq s \leq t$. So, the scalar equation $\dot{x}=\left(-1+\frac{1}{t^{2}+1}\right) x$ has an exponential dichotomy on $\mathbb{R}_{+}$ with exponent $\alpha=-1$.

The difference between the two examples is that the integral of $\frac{1}{t+1}$ in $[0, \infty)$ is divergent whereas the integral of $\frac{1}{t^{2}+1}$ in $[0, \infty)$ is convergent. Thus we guess that that the statement of Theorem 1 can be improved when

$$
\int_{0}^{\infty}|B(t)| d t<\infty
$$

\section{The Main Result}

In this section we prove the following result.

Theorem 3. Suppose the linear system $\dot{x}=A(t) x$ has an exponential dichotomy on $\mathbb{R}_{+}$with exponents $\alpha, \beta$. Then there exists $\varepsilon>0$ such that if $B(t)$ is a piecewise continuous function such that $\sup _{t \in \mathbb{R}_{+}}|B(t)|<\varepsilon$ and

$$
\int_{0}^{\infty}|B(t)| d t<\infty
$$

then the linear system $\dot{x}=[A(t)+B(t)] x$ has an exponential dichotomy on $\mathbb{R}_{+}$with the same exponents $\alpha, \beta$ and projection $Q$ such that

$$
|Q-P|=O(\varepsilon)
$$


A similar result holds when the dichotomies are considered on $\mathbb{R}_{-}$and on $\mathbb{R}$.

Proof. First, replacing $A(t)$ with $A(t)=A(t)+v \mathbb{I}, v=\frac{\alpha-\beta}{2}$, we may assume that the exponents are equal. Denote them by $\delta$. Next, consider the perturbed system

$$
\dot{x}=[A(t)+B(t)] x
$$

Let $\tilde{\delta}<\delta$ and take $\varepsilon>0$ as in Theorem 1. Then (4) has an exponential dichotomy on $\mathbb{R}_{+}$with projection, say, $\tilde{P}$ and exponent $\tilde{\delta}$. We now follow the approach in [10] to construct a suitable projection for the dichotomy of the perturbed equation.

Let $X_{B}(t)$ be the fundamental matrix of system (4) and $X(t)$ be the fundamental matrix of $\dot{x}=A(t) x$. A well known standard argument shows that a bounded solution of (4) satisfies the fixed point equation

$$
\hat{x}(t)=X(t) P \xi+\int_{0}^{t} X(t) P X(s)^{-1} B(s) x(s) d s-\int_{t}^{\infty} X(t)(\mathbb{I}-P) X(s)^{-1} B(s) x(s) d s .
$$

for some $\xi \in \mathbb{R}^{n}$. It is easy to see that if $x(t), x_{1}(t)$ and $x_{2}(t)$ are bounded functions then

$$
|\hat{x}(t)| \leq k|\xi|+\frac{2 k}{\delta}\|B\|\|x\|_{b}
$$

and

$$
\left|\hat{x}_{1}(t)-\hat{x}_{2}(t)\right| \leq \frac{2 k}{\delta}\|B\|\left\|x_{1}-x_{2}\right\|_{b}
$$

So taking $\varepsilon>0$ such that $2 k \varepsilon<\delta$, we see that the map $x(t) \mapsto \hat{x}(t)$ is a uniform contraction (with respect to $\xi)$ on the space $C_{b}^{0}\left(\mathbb{R}_{+}\right)$of bounded continuous functions of $\mathbb{R}_{+}$. So, for any $\xi \in \mathbb{R}^{n}$ the map $x(t) \mapsto \hat{x}(t)$ has a unique fixed point $x(t, \xi)$ such that

$$
\|x(\cdot, \xi)\|_{b} \leq k\left(1-2 k \varepsilon \delta^{-1}\right)^{-1}|\xi| .
$$

Note that $x(t, P \xi)$ is the unique fixed point of

$$
\hat{x}(t)=X(t) P^{2} \xi+\int_{0}^{t} X(t) P X(s)^{-1} B(s) x(s) d s-\int_{t}^{\infty} X(t)(\mathbb{I}-P) X(s)^{-1} B(s) x(s) d s
$$

and then $x(t, P \xi)=x(t, \xi)$, because of $P^{2}=P$ and the uniqueness of the fixed point.

It is straightforward to see that such a fixed point is a solution of (4) and that it is linear with respect to $\xi$. So

$$
x(t, \xi)=X_{B}(t) Q \xi
$$

where

$$
Q \xi=x(0, \xi)=P \xi-\int_{0}^{\infty}(\mathbb{I}-P) X(s)^{-1} B(s) x(s, \xi) d s .
$$

We pause for a moment to observe that

$$
|(Q-P) \xi| \leq \int_{0}^{\infty} k e^{-\delta s}|B(s)||x(s, \xi)| d s \leq k^{2}(\delta-2 k \varepsilon)^{-1} \varepsilon|\xi|
$$

that is

$$
|Q-P| \leq k^{2}(\delta-2 k \varepsilon)^{-1} \varepsilon
$$

From the previous considerations it follows that $X_{B}(t) \xi$ is a bounded solution of (4) if and only if $\xi=Q \xi$. Moreover, we have

$$
\begin{aligned}
& P Q=P \text { and } \\
& Q P \xi=x(0, P \xi)=x(0, \xi)=Q \xi
\end{aligned}
$$


So

$$
Q^{2}=[Q P] Q=Q[P Q]=Q P=Q
$$

that is $Q$ is a projection. Next, if $\xi \in \mathcal{N} Q$ then $P \xi=P Q \xi=0$ and if $\xi \in \mathcal{N} P$ then $Q \xi=Q P \xi=0$. So

$$
\mathcal{N} P=\mathcal{N} Q
$$

Finally, $\xi \in \mathcal{R} Q$ if and only if $X_{B}(t) \xi=X_{B}(t) Q \xi$ is a bounded solution of (4). From Proposition 2 it follows, then, that $Q$ is a projection for the dichotomy of (4). So

$$
\begin{array}{ll}
\left|X_{B}(t) Q X_{B}(s)^{-1}\right| \leq K e^{-\tilde{\delta}(t-s)}, & 0 \leq s \leq t \\
\left|X_{B}(s)(\mathbb{I}-Q) X_{B}(t)^{-1}\right| \leq K e^{-\tilde{\delta}(t-s)}, & 0 \leq s \leq t
\end{array}
$$

for some $K \geq 1$, or, if we go back to the original system with $A(t)$ instead of $A(t)+v \mathbb{I}$ :

$$
\begin{array}{ll}
\left|X_{B}(t) Q X_{B}(s)^{-1}\right| \leq K e^{-\tilde{\alpha}(t-s)}, & 0 \leq s \leq t \\
\left|X_{B}(s)(\mathbb{I}-Q) X_{B}(t)^{-1}\right| \leq K e^{-\tilde{\beta}(t-s)}, & 0 \leq s \leq t .
\end{array}
$$

Now assume that $\int_{0}^{\infty}|B(t)| d t<\infty$ and let $T>0$ be such that

$$
\Delta:=\int_{T}^{\infty}|B(t)| d t<\frac{1}{2 k}
$$

together with $\sup _{t \geq 0}|B(t)| \leq \varepsilon$ and $\alpha=\beta=\delta$. Let $t \geq s \geq T$. From the previous part we know that $x(t, s, \xi)=X_{B}(t) Q X_{B}(s)^{-1} \xi$ is a solution of $\dot{x}=[A(t)+B(t)] x$ which is bounded for $t \geq s \geq T$. Actually we have

$$
|x(t, s, \xi)| \leq k|\xi| e^{-\tilde{\delta}(t-s)} .
$$

We want to show that $\tilde{\delta}$ can be replaced by $\delta$. To this end we consider the map $x(t) \mapsto \hat{x}(t)$ :

$$
\begin{aligned}
\hat{x}(t) & =X(t) P X(s)^{-1} \xi+\int_{s}^{t} X(t) P X(\sigma)^{-1} B(\sigma) x(\sigma) d \sigma \\
& -\int_{t}^{\infty} X(t)(\mathbb{I}-P) X(\sigma)^{-1} B(\sigma) x(\sigma) d s
\end{aligned}
$$

in the space $C_{\delta}^{0}([s, \infty)), s \geq T$, of functions $x(t)$ such that

$$
\sup _{t \geq s}|x(t)| e^{\delta(t-s)}<\infty
$$

with norm $\|x(\cdot)\|=\sup _{t \geq s}|x(t)| e^{\delta(t-s)}$. We have

$$
\begin{aligned}
& |\hat{x}(t)| \leq k e^{-\delta(t-s)}|\xi|+\int_{s}^{t} k e^{-\delta(t-s)}|B(\sigma)|\|x(\cdot)\| d s+\int_{t}^{\infty} k e^{\delta(t+s-2 \sigma)}|B(\sigma)|\|x(\cdot)\| d s \\
& \leq k e^{-\delta(t-s)}\left(|\xi|+\int_{s}^{\infty}|B(\sigma)| d \sigma\|x(\cdot)\|\right) \leq k e^{-\delta(t-s)}(|\xi|+\Delta\|x(\cdot)\|) .
\end{aligned}
$$

or else

$$
\|\hat{x}(\cdot)\| \leq k(|\xi|+\Delta\|x(\cdot)\|)
$$

and similarly

$$
\left\|\hat{x}_{1}(\cdot)-\hat{x}_{2}(\cdot)\right\| \leq k \Delta\left\|x_{1}(\cdot)-x_{2}(\cdot)\right\| .
$$

So we have proved the following 
Proposition 3. Suppose the linear system (1) has an exponential dichotomy on $\mathbb{R}_{+}$with projection P constant $k$ and exponents $\alpha=\beta=\delta$. Let $B(t)$ be a matrix and suppose there exists $T \geq 0$ such that such that

$$
\|B\|=\sup _{t \geq T}|B(t)| \leq \varepsilon
$$

and

$$
\int_{T}^{\infty}|B(t)| d t=\Delta<\infty
$$

where $\varepsilon>0$ is sufficiently small and $\Delta$ satisfies $2 k \Delta \leq 1$. Then for any $s \geq T$ the map (7) is a contraction on the set $C_{\delta}^{0}([s, \infty))$ and contraction constant $=\frac{1}{2}$. Thus its unique fixed point $x(t, s, \xi)$ belongs to $C_{\delta}^{0}([s, \infty))$ and

$$
\|x(t, s, \xi)\| \leq 2 k|\xi|
$$

Hence we proved that

$$
\left|X_{B}(t) Q X_{B}(s)^{-1}\right| \leq 2 k e^{-\delta(t-s)}
$$

for any $t \geq s \geq T$, and we extend this inequality for any $t \geq s \geq 0$ provided we change $2 k$ with a possibly larger constant $K_{1}$. Next, from Proposition 2, we also know that

$$
\left|X_{B}(s)(\mathbb{I}-Q) X_{B}(t)^{-1}\right| \leq K_{2} e^{-\tilde{\delta}(t-s)}
$$

for $0 \leq s \leq t$ and possibly another constant $K_{2}$, since we know that $Q$ can be taken as a projection of the dichotomy of the perturbed system. Thus:

$$
\begin{aligned}
& \left|X_{B}(t) Q X_{B}(s)^{-1}\right| \leq K e^{-\delta(t-s)} \\
& \left|X_{B}(s)(\mathbb{I}-Q) X_{B}(t)^{-1}\right| \leq K e^{-\tilde{\delta}(t-s)}
\end{aligned}
$$

(where $\tilde{\delta}<\delta$ ) for any $t \geq s \geq 0$ and $K=\max \left\{K_{1}, K_{2}\right\}$.

To complete the proof we still have to prove that, for $T \leq s \leq t$, it results

$$
\left|X_{B}(s)(\mathbb{I}-Q) X_{B}(t)^{-1}\right| \leq K e^{-\delta(t-s)}
$$

for possibly another constant $K$. The fundamental matrix $Y(t)=X(t)^{-1 *}$ of the adjoint system

$$
\dot{x}=-A(t)^{*} x
$$

has an exponential dichotomy on $\mathbb{R}_{+}$with projection $\left(\mathbb{I}-P^{*}\right)$. Indeed:

$$
\begin{aligned}
& \left|Y(t)\left(\mathbb{I}-P^{*}\right) Y(s)^{-1}\right| \leq k e^{-\delta(t-s)} \\
& \left|Y(t) P^{*} Y(s)^{-1}\right| \leq k e^{-\delta(t-s)}
\end{aligned}
$$

for any $0 \leq s \leq t$. From the previous part applied to the system $\dot{x}=-[A(t)+B(t)]^{*} x$ we see that a projection $\tilde{Q}^{*}$ exists such that $\mathcal{R} \tilde{Q}^{*}=\mathcal{R} P^{*}$ and

$$
\begin{aligned}
& \left|Y_{B}(t)\left(\mathbb{I}-\tilde{Q}^{*}\right) Y_{B}(s)^{-1}\right| \leq K e^{-\delta(t-s)} \\
& \left|Y_{B}(s) \tilde{Q}^{*} Y_{B}(t)^{-1}\right| \leq K e^{-\tilde{\delta}(t-s)}
\end{aligned}
$$

for $0 \leq s \leq t$, where $Y_{B}(t)=X_{B}(t)^{-1 *}$ is the fundamental matrix of the perturbed system $\dot{x}=$ $-[A(t)+B(t)]^{*} x$. Going back to $X_{B}(t)$ we see that

$$
\begin{aligned}
& \left|X_{B}(t) \tilde{Q} X_{B}(s)^{-1}\right| \leq K e^{-\tilde{\delta}(t-s)} \\
& \left|X_{B}(s)(\mathbb{I}-\tilde{Q}) X_{B}(t)^{-1}\right| \leq K e^{-\delta(t-s)}
\end{aligned}
$$


for $0 \leq s \leq t$ and a certain constant $K$ (possibly different from the previous one, however we do not introduce other notations for these constants since at the end we can take the larger of all). From the first inequality it follows that, if $\xi \in \mathcal{R} \widetilde{Q}$ then $\left|X_{B}(t) \xi\right| \leq K e^{-\tilde{\delta} t}$ and hence $\mathcal{R} \tilde{Q} \subset \mathcal{R} Q$. So

$$
\mathcal{R} \widetilde{Q}=\mathcal{R} Q
$$

since $\operatorname{rank} \tilde{Q}=\operatorname{rank} \tilde{Q}^{*}=\operatorname{rank} P^{*}=\operatorname{rank} P=\operatorname{rank} Q$. Next:

$$
\begin{aligned}
& \xi \in \mathcal{N} \tilde{Q} \Leftrightarrow \tilde{Q} \xi=0 \Leftrightarrow\langle\tilde{Q} \xi, \eta\rangle=0, \forall \eta \\
& \Leftrightarrow\left\langle\xi, \tilde{Q}^{*} \eta\right\rangle=0, \forall \eta \Leftrightarrow \xi \in\left[\mathcal{R} \tilde{Q}^{*}\right]^{\perp}=\left[\mathcal{R} P^{*}\right]^{\perp} .
\end{aligned}
$$

But in the same way we see that $\left[\mathcal{R} P^{*}\right]^{\perp}=\mathcal{N} P$ and hence

$$
\mathcal{N} \tilde{Q}=\mathcal{N} P=\mathcal{N} Q .
$$

As a consequence $Q=\tilde{Q}$ and we have

$$
\left|X_{B}(t) Q X_{B}(s)^{-1}\right| \leq K e^{-\delta(t-s)}
$$

and

$$
\left|X_{B}(s)(\mathbb{I}-Q) X_{B}(t)^{-1}\right|=\left|X_{B}(s)(\mathbb{I}-\tilde{Q}) X_{B}(t)^{-1}\right| \leq K e^{-\delta(t-s)}
$$

for $0 \leq s \leq t$

Going back to the original system (that is before the shifting from $A(t)$ to $A(t)+v \mathbb{I}$ ) we see that

$$
\begin{aligned}
& \left|X_{B}(t) Q X_{B}(s)^{-1}\right| \leq 2 K e^{-\alpha(t-s)} \\
& \left|X_{B}(s)(\mathbb{I}-Q) X_{B}(t)^{-1}\right| \leq 2 K e^{-\beta(t-s)} .
\end{aligned}
$$

for $0 \leq s \leq t$. This completes the proof when the dichotomy is on $\mathbb{R}_{+}$.

When the dichotomy is on $\mathbb{R}_{-}$, we reduce to the case of $\mathbb{R}_{+}$by changing $t$ with $-t, X(t)$ with $X(-t)$ and $A(t)$ with $-A(-t)$. When $\dot{x}=A(t) x$ has an exponential dichotomy on $\mathbb{R}$, we apply the previous result to see that $\dot{x}=[A(t)+B(t)] x$ has an exponential dichotomy on $\mathbb{R}_{+}$with projection $Q_{+}$ and on $\mathbb{R}_{-}$with projection $Q_{-}$. Then $\mathcal{R} Q_{+} \cap \mathcal{N} Q_{-}=\{0\}$ because both projections are close to $P$ and $\mathcal{R} P \cap \mathcal{N} P=\{0\}$ since $\dot{x}=A(t) x$ has an exponential dichotomy on $\mathbb{R}$. The conclusion follows from [3] [p. 19], (see also [2] (Proposition 2.1)).

\section{Asymptotically Constant Matrices}

Let $A(t)$ be a piecewise continuous $n \times n$ matrix, $t \in \mathbb{R}_{+}$and assume that a constant matrix $A$ exists such that

(A1) $\lim _{t \rightarrow \infty} A(t)=A$ and $\int_{0}^{\infty}|A(t)-A| d t<\infty$;

(A2) $A$ has two semi-simple eigenvalues $-\alpha<0$ and $\beta>0$;

(A3) there exists $\mu>0$ such that all others eigenvalues $\lambda$ of $A$ satisfy either $\Re \lambda \leq-(\alpha+\mu)$ or $\Re \lambda \geq \beta+\mu$.

Let $X_{0}(t)$ be the fundamental matrix of $\dot{x}=A x$ such that $X_{0}(0)=\mathbb{I}$. Since $-\alpha$ and $\beta$ are semi-simple eigenvalues, their generalized eigenspaces, that we denote with $V_{s}$ and $V_{u}$, consist of eigenvectors of $-\alpha$ and $\beta$, that is for any $v \in V_{s}$ (resp. $v \in V_{u}$ ) we have $X_{0}(t) v=v e^{-\alpha t}$ (resp. $\left.X_{0}(t) v=v e^{\beta t}\right)$. Write

$$
\mathbb{R}^{n}=V_{s s} \oplus V_{s} \oplus V_{u} \oplus V_{u u}
$$

where $V_{s s}$ is the generalized eigenspace of the eigenvalues of $A$ with real parts less than $-\alpha-\mu$ and $V_{u u}$ is the generalized eigenspace of the eigenvalues of $A$ with real parts greater than $\alpha+\mu$. Let $d_{s s}, d_{s}$, $d_{u} d_{u u}$ be the dimensions of $V_{s s}, V_{s} V_{u}, V_{u u}$ respectively. 
Let $P_{s s}: \mathbb{R}^{n} \rightarrow \mathbb{R}^{n}$ be the projection onto $V_{s s}$ with kernel $V_{s} \oplus V_{u} \oplus V_{u u}, P_{s}: \mathbb{R}^{n} \rightarrow \mathbb{R}^{n}$ be the projection onto $V_{s}$ with kernel $V_{s s} \oplus V_{u} \oplus V_{u u}, P_{u}: \mathbb{R}^{n} \rightarrow \mathbb{R}^{n}$ be the projection onto $V_{u}$ with kernel $V_{s s} \oplus V_{s} \oplus V_{u u}$, and $P_{u u}: \mathbb{R}^{n} \rightarrow \mathbb{R}^{n}$ be the projection onto $V_{u u}$ with kernel $V_{s s} \oplus V_{s} \oplus V_{u}$.

Let $\left\{v_{1}^{s S}, \ldots, v_{d_{S S}^{S S}}^{S S}\right.$ be a orthonormal basis of $V_{s S},\left\{v_{1}^{S}, \ldots, v_{d_{s}}^{S}\right\}$ be a orthonormal basis of $V_{S}$, $\left\{v_{1}^{u}, \ldots, v_{d_{u}}^{u}\right\}$ be a orthonormal basis of $V_{u}$ and $\left\{v_{1}^{u u}, \ldots, v_{d_{u u}}^{u u}\right\}$ be a orthonormal basis of $V_{u u}$. For any $\xi \in \mathbb{R}^{n}$ we have

$$
\begin{array}{ll}
P_{s s} \xi=\sum_{j=1}^{d_{s s}} c_{j}^{s s} v_{j}^{s S} & P_{s} \xi=\sum_{j=1}^{d_{s}} c_{j}^{s} v_{j}^{s} \\
P_{u u} \xi=\sum_{j=1}^{d_{u u}} c_{j}^{u u} v_{j}^{u u} & P_{u} \xi=\sum_{j=1}^{d_{u}} c_{j}^{u} v_{j}^{u}
\end{array}
$$

Hence

$$
\left\{\sum_{j=1}^{d_{s S}}\left|c_{j}^{s S}\right|^{2}\right\}^{\frac{1}{2}}=\left|P_{s s} \xi\right| \leq\left|P_{s s}\right||\xi| .
$$

Similarly

$$
\begin{aligned}
& \left\{\sum_{j=1}^{d_{s}}\left|c_{j}^{s}\right|^{2}\right\}^{\frac{1}{2}} \leq\left|P_{s}\right||\xi| \\
& \left\{\sum_{j=1}^{d_{u}}\left|c_{j}^{u}\right|^{2}\right\}^{\frac{1}{2}} \leq\left|P_{u}\right||\xi| \\
& \left\{\sum_{j=1}^{d_{u u}}\left|c_{j}^{u u}\right|^{2}\right\}^{\frac{1}{2}} \leq\left|P_{u u}\right||\xi| .
\end{aligned}
$$

Next, $V_{s s}, V_{s}, V_{u}$ and $V_{u u}$ are all invariant under $X_{0}(s)$, that is

$$
\xi \in V \Rightarrow X_{0}(s) \xi \in V
$$

for $V=V_{s s}, V_{s}, V_{u}, V_{u u}$. So we have, for example

$$
X_{0}(t) P_{s s} \xi=P_{s s} X_{0}(t) P_{s s} \xi
$$

and

$$
P_{s s} X_{0}(t)\left(\mathbb{I}-P_{s s}\right)=0
$$

because $X_{0}(t)\left(\mathbb{I}-P_{s s}\right)=X_{0}(t) P_{s}+X_{0}(t) P_{u}+X_{0}(t) P_{u u} \in V_{s}+V_{u}+V_{u u}$. So

$$
X_{0}(t) P_{s S}=P_{s S} X_{0}(t) .
$$

Similarly:

$$
\begin{aligned}
& X_{0}(t) P_{s}=P_{s} X_{0}(t) \\
& X_{0}(t) P_{u}=P_{u} X_{0}(t) \\
& X_{0}(t) P_{u u}=P_{u u} X_{0}(t) .
\end{aligned}
$$

Now we observe that

$$
\begin{aligned}
& X_{0}(t) X_{0}(s)^{-1} P_{s} \xi=X_{0}(t-s) P_{s} \xi=X_{0}(t-s) \sum_{j=1}^{d_{s}} c_{j}^{s} v_{j}^{s}=\sum_{j=1}^{d_{s}} c_{j}^{s} X_{0}(t-s) v_{j}^{s} \\
& =e^{-\alpha(t-s)} \sum_{j=1}^{d_{s}} c_{j}^{s} v_{j}^{s}=e^{-\alpha(t-s)} P_{s} \xi
\end{aligned}
$$


then

$$
\left|X_{0}(t) X_{0}(s)^{-1} P_{s}\right| \leq\left|P_{s}\right| e^{-\alpha(t-s)}
$$

for any $0 \leq s, t$. Similarly,

$$
\left|X_{0}(t) X_{0}(s)^{-1} P_{u}\right| \leq\left|P_{u}\right| e^{\beta(t-s)}
$$

for any $0 \leq s, t$. A slightly different estimate occurs when considering $Y_{0}(t) Y_{0}(s)^{-1} P_{s s}$ and $Y_{0}(t) Y_{0}(s)^{-1} P_{u u}$. Indeed in this case the eigenvalues may not be simple so that, for example

$$
X_{0}(t) X_{0}(s)^{-1} v_{i}^{s s}=X_{0}(t-s) v_{i}^{s s}=\sum_{j=1}^{d_{s s}} q_{i j}(t-s) e^{\lambda_{j}(t-s)} v_{j}^{s s}
$$

where $q_{i j}(t)$ is a polynomial that may have positive degree (but less than the multiplicity of $\lambda_{i}$ as an eigenvalue of $A$.) Since $\Re \lambda_{i} \leq-\alpha-\mu$, for any $i=1, \ldots, d_{S S}$ in this case we have then

$$
\left|X_{0}(t) X_{0}(s)^{-1} v_{i}^{s S}\right|=\left|X_{0}(t-s) v_{i}^{s \mathcal{S}}\right| \leq c_{i} e^{-\left(\alpha+\frac{\mu}{2}\right)(t-s)}
$$

for some $c_{i}>0$. As a consequence

$$
\begin{aligned}
& \left|X_{0}(t) X_{0}(s)^{-1} P_{S s} \xi\right| \leq \sum_{i=1}^{d_{s s}}\left|c_{i}^{s S}\right|\left|X_{0}(t-s) v_{i}^{s S}\right| \leq c_{i} \sum_{i=1}^{d_{s s}}\left|c_{i}^{s S}\right| e^{-\left(\alpha+\frac{\mu}{2}\right)(t-s)} \\
& \leq k_{1}\left|P_{s S}\right| e^{-\left(\alpha+\frac{\mu}{2}\right)(t-s)}|\xi|
\end{aligned}
$$

for any $t \geq s \geq 0$. Similarly:

$$
\left|X_{0}(t) X_{0}(s)^{-1} P_{u u} \xi\right| \leq k_{2}\left|P_{u u}\right| e^{\left(\beta+\frac{\mu}{2}\right)(t-s)}|\xi|
$$

for some $k_{2}$ and any $s \geq t \geq 0$. Summarising we see that $k \geq 1$ exists such that:

$$
\begin{array}{ll}
\left|X_{0}(t) X_{0}(s)^{-1} P_{s s}\right| \leq k\left|P_{s s}\right| e^{-\left(\alpha+\frac{\mu}{2}\right)(t-s)} & \text { for any } 0 \leq s \leq t \\
\left|X_{0}(t) X_{0}(s)^{-1} P_{s}\right| \leq\left|P_{s}\right| e^{-\alpha(t-s)} & \text { for any } 0 \leq s, t \\
\left|X_{0}(t) X_{0}(s)^{-1} P_{u}\right| \leq\left|P_{u}\right| e^{\beta(t-s)} & \text { for any } 0 \leq s, t \\
\left|X_{0}(t) X_{0}(s)^{-1} P_{u u}\right| \leq k\left|P_{u u}\right| e^{(\beta+f r a c \mu 2)(t-s)} & \text { for any } 0 \leq t \leq s .
\end{array}
$$

and hence, using the commutativity of $X_{0}(s)$ with the projections

$$
\begin{array}{ll}
\left|X_{0}(t) P_{s s} X_{0}(s)^{-1}\right| \leq k\left|P_{s s}\right| e^{-\left(\alpha+\frac{\mu}{2}\right)(t-s)} & \text { for any } 0 \leq s \leq t \\
\left|X_{0}(t) P_{s} X_{0}(s)^{-1}\right| \leq\left|P_{s}\right| e^{-\alpha(t-s)} & \text { for any } 0 \leq s, t \\
\left|X_{0}(t) P_{u} X_{0}(s)^{-1}\right| \leq\left|P_{u}\right| e^{\beta(t-s)} & \text { for any } 0 \leq s, t \\
\left|X_{0}(t) P_{u u} X_{0}(s)^{-1}\right| \leq k\left|P_{u u}\right| e^{\left(\beta+\frac{\mu}{2}\right)(t-s)} & \text { for any } 0 \leq t \leq s .
\end{array}
$$

Setting

$$
P=P_{S S}+P_{S}
$$

and then $I-P=P_{u u}+P_{u}$ we get

$$
\begin{array}{ll}
\left|X_{0}(t) P X_{0}(s)^{-1}\right| \leq\left(\left|P_{s}\right|+k\left|P_{s s}\right|\right) e^{-\alpha(t-s)} & \text { for any } t \geq s \geq 0 \\
\left|X_{0}(s)(\mathbb{I}-P) X_{0}(t)^{-1}\right| \leq\left(\left|P_{u}\right|+k\left|P_{u u}\right|\right) e^{\beta(t-s)} & \text { for any } s \geq t \geq 0
\end{array}
$$

From Theorem 3 we obtain the following result.

Proposition 4. Suppose conditions (A1)-(A3) hold. Then the linear system $\dot{x}=A(t) x$ has an exponential dichotomy on both $\mathbb{R}_{+}$and $\mathbb{R}_{-}$with exponents $\alpha$ and $\beta$. 
We conclude this Section with an application of Proposition 4 to nonlinear systems. Let $f(x)$ be a $C^{1}$-function such that $f^{\prime}(x)$ is Lipschitz with $L_{f}$ as Lipschitz constant. Suppose the system $\dot{x}=f(x)$ has two hyperbolic fixed points $x=x_{-}$and $x_{+}$(that may coincide, i.e., $x_{-}=x_{+}$) together with a heteroclinic orbit $u(t)$, i.e., a bounded solution such that

$$
\lim _{t \rightarrow \pm \infty} u(t)=x_{ \pm}
$$

The fixed points being hyperbolic means that the matrices $f^{\prime}\left(x_{-}\right)$and $f^{\prime}\left(x_{+}\right)$have no eigenvalues with zero real part. Then both systems

$$
\dot{x}=f^{\prime}\left(x_{ \pm}\right) x
$$

have an exponential dichotomy on $\mathbb{R}$ with projections, say, $P_{+}$and $P_{-}$. It is known that rank $P_{ \pm}$equals the number of eigenvalues of $f^{\prime}\left(x_{ \pm}\right)$having negative real parts counted with multiplicities. Let $\alpha_{ \pm}$and $\beta_{ \pm}$be the exponents of the dichotomy of $\dot{x}=f^{\prime}\left(x_{ \pm}\right) x$ respectively. First we observe that $\dot{u}(t)=f(u(t))$ is bounded and it is also a solution of $\dot{x}=f^{\prime}(u(t)) x$. From the roughness theorem we know that this system has an exponential dichotomy on $\mathbb{R}_{+}$with exponents $\tilde{\alpha}_{+}$and $\tilde{\beta}_{+}$slightly less that $\alpha_{+}$and $\beta_{+}$respectively. Hence we get $\left|u(t)-x_{+}\right| \leq K e^{-\tilde{\alpha}_{+} t}$ for $t \geq 0$. Similarly we get $\left|u(t)-x_{-}\right| \leq K e^{\tilde{\beta}_{-} t}$, for $t \leq 0$. So we see that $f^{\prime}(u(t))-f^{\prime}\left(x_{+}\right) \in L^{1}\left(\mathbb{R}_{+}\right)$and $f^{\prime}(u(t))-f^{\prime}\left(x_{-}\right) \in L^{1}\left(\mathbb{R}_{-}\right)$. A simple application of Theorem 3 gives then the following

Theorem 4. Let $f(x)$ be a $C^{1}$-function with Lipschitz continuous derivative. Suppose there exists $x=x_{-}$ and $x_{+}$such that $f\left(x_{-}\right)=f\left(x_{+}\right)=0$ and $f^{\prime}\left(x_{-}\right), f^{\prime}\left(x_{+}\right)$has no eigenvalues with zero real parts. Then both linear systems $\dot{x}=f^{\prime}\left(x_{-}\right) x$ and $\dot{x}=f^{\prime}\left(x_{+}\right) x$ have an exponential dichotomy on $\mathbb{R}$. Let $\alpha_{ \pm}, \beta_{ \pm}$and $P_{ \pm}$be the corresponding exponents and projections. Suppose further that the (nonlinear) equation $\dot{x}=f(x)$ has a solution $u(t)$ such that

$$
\lim _{t \rightarrow \pm \infty} u(t)=x_{ \pm}
$$

Then the linear equation $\dot{x}=f^{\prime}(u(t)) x$ has an exponential dichotomy on both $\mathbb{R}_{-}$and $\mathbb{R}_{+}$with exponents $\alpha_{-}, \beta_{-}$and $\alpha_{+}, \beta_{+}$respectively, and projections $Q_{ \pm}$such that

$$
\operatorname{rank} Q_{ \pm}=\operatorname{rank} P_{ \pm}
$$

\section{Conclusions}

We have given a new proof of a roughness result for linear systems with an exponential dichotomy different than the one in [8]. This new proof has the advantage that it is is more direct, can be easily extended to system having an exponential dichotomy on the whole line and gives a precise estimate on the norm of the difference of the projections of the dichotomies of the perturbed and the unperturbed system. Moreover it extends also to more general situations. Indeed the assumptions that $\sup _{t \in I}|B(t)|<\varepsilon$ is used just to prove that the map

$$
x(t) \mapsto X(t) P \xi+\int_{0}^{\infty} \Gamma(t, s) B(s) x(s) d s,
$$

where

$$
\Gamma(t, s)= \begin{cases}X(t) P X(s)^{-1} & \text { if } 0 \leq s \leq t \\ -X(t)(\mathbb{I}-P) X(s)^{-1} & \text { if } 0 \leq t<s,\end{cases}
$$

is a contraction on $C_{b}^{0}$. According to [7] this holds also under the weaker assumption that

$$
\inf _{T>0} \sup _{t \geq 0} \int_{t}^{t+T}|B(s)| d s\left(\frac{k}{1-e^{-\alpha T}}+\frac{k}{1-e^{-\beta T}}\right)<1
$$


and the fixed point $x(t, s)$ satisfies again $\|x\| \leq C|\xi|$, for a suitable constant $C$. The remaining part of the proof showing that this fixed point indeed belongs to $C_{\delta}^{0}$ just depends on the fact that $|B(t)| \in L^{1}\left(\mathbb{R}_{+}\right)$. Hence Theorem 3 holds also under the weaker condition (10) instead of $\sup _{t \geq 0}|B(t)|<\varepsilon$.

Author Contributions: Investigation, M.F.; Methodology, F.B. The contributions of all authors are equal. All authors have read and agreed to the published version of the manuscript .

Funding: Partially supported by the Slovak Research and Development Agency under the contract No. APVV-18-0308 and by the Slovak Grant Agency VEGA No. 1/0358/20 and No. 2/0127/20.

Conflicts of Interest: The authors declare no conflict of interest.

\section{References}

1. Perron, O. Die Stabilitätsfrage bei Differentialgleichungen. Math. Z. 1930, 32, 703-728. [CrossRef]

2. Palmer, K.J. Exponential dichotomies and transversal homoclinic points. J. Diff. Equ. 1984, 55, 225-256. [CrossRef]

3. Coppel, W.A. Dichotomies in Stability Theory; Lecture Notes in Mathematics 629; Springer: Berlin/Heidelberg, Germany, 1978.

4. Palmer, K.J. Exponential dichotomy, integral separation and diagonalizability of linear systems of ordinary differential equations. J. Diff. Equ. 1982, 43, 184-203. [CrossRef]

5. Palmer, K.J. Exponential dichotomy, exponential separation and spectral theory for linear systems of ordinary differential equations. J. Diff. Equ. 1982, 46, 324-345. [CrossRef]

6. Battelli, F.; Palmer, K.J. Strongly exponentially separated linear systems. J. Dyn. Diff. Equ. 2019, 31, 573-600. [CrossRef]

7. Ju, N.; Wiggins, S. On roughness of exponential dichotomy. J. Math. Anal. Appl. 2001, 262, 39-49. [CrossRef]

8. Calamai, A.; Franca, M. Mel'nikov methods and homoclinic orbits in discontinuous systems. J. Dyn. Diff. Equ. 2013, 25, 733-764. [CrossRef]

9. Battelli, F.; Fečkan, M. Melnikov theory for nonlinear implicit ODEs. J. Diff. Equ. 2014, 256, 1157-1190. [CrossRef]

10. Hale, J. Introductions to dynamic bifurcation. In Bifurcation Theory and Applications; Lecture Notes in Mathematics 1057; Springer: Berlin, Germany, 1984; pp. 106-151.

(C) 2020 by the authors. Licensee MDPI, Basel, Switzerland. This article is an open access article distributed under the terms and conditions of the Creative Commons Attribution (CC BY) license (http://creativecommons.org/licenses/by/4.0/). 